\title{
A retrospective study on the evaluation of the symptoms, medications and improvement of the quality of life of patients undergoing robotic surgery for gastroesophageal reflux disease
}

\author{
SOFIA K. KONSTANTINIDOU ${ }^{1}$, PENELOPE KOSTARAS ${ }^{2}$, GEORGIOS-ELEFTHERIOS ANAGNOSTOPOULOS ${ }^{2}$, \\ SOPHIA-LIBERTY MARKANTONIS ${ }^{2}$, VANGELIS KARALIS ${ }^{2}$ and KONSTANTINOS KONSTANTINIDIS ${ }^{3}$ \\ ${ }^{1}$ Clinical Pharmacy Department, Athens Medical Center, 15125 Athens; \\ ${ }^{2}$ Department of Pharmacy, School of Health Sciences, National and Kapodistrian University of Athens, 15784 Athens; \\ ${ }^{3}$ Department of General, Laparoscopic, Robotic and Bariatric Surgery, Athens Medical Center, 15125 Athens, Greece
}

Received November 20, 2020; Accepted December 10, 2020

DOI: $10.3892 / \mathrm{etm} .2020 .9605$

\begin{abstract}
Gastroesophageal reflux disease (GERD) is a common gastrointestinal disorder requiring lifestyle adaptations and administration of medications. Another approach is the surgical treatment of GERD through laparoscopic or robotic operations. The aim of the present study was to investigate the improvement of symptoms and quality of life of patients with GERD, before and after robotic surgical restoration using the Nissen robotic fundoplication technique. The potential effects of body weight, age and sex, as well as the response to medications and progress over time, were also assessed. A retrospective study was conducted in a tertiary hospital between October 2019 and March 2020. Data were collected and recorded from 144 patients who underwent robotic surgery, using the Nissen fundoplication technique, during the period 2009-2019. All patients involved in this analysis pre-operatively exhibited severe symptoms of heartburn and reflux, as well as poor quality of life. All of these symptoms were re-examined after surgery, and a marked decrease was observed with respect to their frequency and intensity. Improvement was not affected by body mass index, whereas older patients exhibited greater improvement. Women initially experienced more severe symptoms before the surgery, but they appeared to respond as well as the male patients. The long-term beneficial effects of surgery for up to the 10 -year period studied were validated. After the robotic surgical rehabilitation, the vast majority of patients overcame the unpleasant symptoms of GERD and stayed off their
\end{abstract}

Correspondence to: Professor Vangelis Karalis, Department of Pharmacy, School of Health Sciences, National and Kapodistrian University of Athens, University Campus, Pharmacy building, 15784 Athens, Greece

E-mail: vkaralis@pharm.uoa.gr

Key words: gastroesophageal reflux disease, robotic surgery, quality of life, retrospective study, statistical analysis medications. More than $4 / 5$ of the patients were satisfied after surgery. In conclusion, restoration of GERD, using Nissen robotic fundoplication, led to the minimization of symptoms and to a marked improvement in the quality of life of patients.

\section{Introduction}

Gastroesophageal reflux disease (GERD) is a very common disorder that affects the lower esophageal sphincter, the muscle between the esophagus and stomach, which acts as a barrier against reflux (1). Esophageal peristalsis is important against reflux because it is the main factor of esophageal cleansing, whereas dysfunctional peristalsis is associated with severe GERD (2). GERD is widespread; population-based studies have reported that $18-27 \%$ of the North American population, 8-25\% of Europeans, $2-7 \%$ of East Asians and 23\% of South Americans suffer from GERD (3).

The most common symptom of GERD is heartburn, and other symptoms include swallowing difficulties and reflux $(1,4,5)$. In addition, acid reflux can cause bronchospasm, which can worsen underlying asthma, leading to coughing, shortness of breath and wheezing (6). Some patients with GERD may also experience nausea and vomiting. It is important to examine patients for symptoms associated with underlying malignancy, accompanied by symptoms such as dysphagia, painful swallowing, anemia, bleeding and weight loss. If left untreated, GERD can cause serious complications, such as esophagitis and Barrett's esophagus $(7,8)$.

For diagnosis, the first step is to record patient history to verify the symptoms, their duration, intensity and association with specific foods, posture and exercise (5). If symptoms are temporary, empirical treatment is provided in order to inhibit acid secretion. The improvement of symptoms confirms the clinical existence of GERD. If the empirical treatment does not improve symptoms, methods such as endoscopy, esophageal pH monitoring, esophageal manometry and barium esophagogram are used to detect secondary mucosal complications (7).

There are some lifestyle changes that can reduce the intensity of symptoms, including smoking cessation, reduction of alcohol consumption, weight loss and particularly 
the reduction of abdominal obesity, avoidance of fatty foods and coffee consumption, and avoidance of eating late-night meals (5,8-16). Posture during night sleep also appears to have a significant role in the improvement of symptoms; a bed head higher than 15-20 cm appears the least effective (1). If the symptoms persist, despite the lifestyle modifications, patients can be treated with medicines, such as antacids, $\mathrm{H}_{2}$-histamine receptor antagonists and proton pump inhibitors (PPIs). PPIs are the most successful pharmaceutical treatment against GERD since they can cause rapid relief of symptoms (17). A first-line treatment for the improvement of GERD is the use of PPIs for 8 weeks (15). However, studies have demonstrated that long-term and uncontrolled use of PPIs has risks, including kidney damage, reduced absorption of nutrients (e.g., B12 and calcium), dementia and intestinal infections by Clostridium difficile; it can also be a risk factor for stomach cancer (17-21).

In the last two decades, laparoscopic and robotic surgical techniques have been employed in the treatment of GERD. Robotic surgery is a minimally invasive method that combines the capabilities of open surgery with the advantages of laparoscopic surgery. Such advantages of robotic surgery refer to the high degree of freedom because of robotic arms, the visual contact of the surgical field with a real 3D projection, and the huge improvements in stapling and stitching, thanks to the articulated parts of the robotic machine (22).

The aim of the present retrospective study was to evaluate the improvement in symptoms and quality of life of patients undergoing robotic surgery for GERD. More specifically, the quality of life of patients was compared pre-operatively (when they received medication to treat symptoms) and post-operatively. GERD symptoms, such as heartburn, dysphagia and reflux, as well as response to medications, patient satisfaction, total score for GERD, demographics and habits, were collected and analyzed. The potential effects of body weight, age and sex were also assessed in the analysis. Finally, the medications received for treating GERD symptoms were evaluated both before and after the surgery.

\section{Materials and methods}

Clinical unit. The present study was conducted at the Department of General,-Laparoscopic, Bariatric, Robotic Surgery, Athens Medical Center (Athens, Greece), between October 2019 and March 2020, after ethics approval by the Scientific Committee of the Athens Medical Center. The analysis compiled data from 144 patients who underwent surgery to treat GERD symptoms between 2009 and 2019. For all patients studied, robotic surgery was applied using the Nissen fundoplication technique $(23,24)$.

Data organization and questionnaire encoding. Initially, the basic information about patients (demographics, habits and surgery records) was received from the electronic records of the hospital. After personal communication with patients, a questionnaire was completed (25). By completing the questionnaire, pre- and post-operative information and personal experiences were recorded. The questions included queries about symptoms before surgery and the medication they were receiving; the same questions were repeated post-operatively. More specifically, patients were asked to rate their symptoms on a scale of
0-5 as follows: 0 , no symptoms; 1 , perceived symptom but not bothering; 2, perceived symptom bothering, but not every day; 3 , symptoms annoying every day; 4 , symptoms that affect daily activity; 5, symptoms that interfere with daily activity.

In order to assess any improvement in symptoms due to surgery, the frequency and intensity of symptoms for each patient post-operatively were compared with those pre-operatively. In addition, patients were asked to provide their opinion directly on the extent of improvement by grading according to the system of 'no improvement', 'intermediate' and 'great improvement'. In addition, based on the individual symptoms score (for several types of reflux, swallowing, and heartburn), the total scores for reflux and heartburn, as well as the total GERD score, were calculated (25).

Quality of life was assessed in two ways: i) Based on the total score of the aforementioned questionnaire, where the higher the score value, the worse the quality of life; ii) by asking the patients directly if they were satisfied with their situation with possible answers yes/no/neutral. In addition, in an effort to assess the effects of surgery over time, patients were divided into three categories. The first contained individuals who had undergone surgery in the last 2 years (42 patients), the second category of patients had undergone surgery within 5 years (49 patients), and the third category of patients had undergone surgery up to 10 years ago (53 patients) (26). Based on this categorization, the possibility of reflux recurrence and heartburn over time was assessed.

Statistical analysis. Data collection was followed by statistical processing using Excel (version 16.43 for macOS) software (Microsoft Corporation) and the IBM $^{\circledR}$ SPSS $^{\circledR}$ statistical platform (version 25; IBM Corp.). The analysis included descriptive statistics and statistical comparisons among groups.

Descriptive statistics. Initially, descriptive statistics were applied to summarize the data, and provide an overview of the trends and differences. Based on the patient scores in the questionnaire, diagrams were developed for each symptom before and after surgery. In addition, with the aim of investigating the role of BMI, sex and age group ( $<55$ or $\geq 55$ years), data were also analyzed in terms of these factors.

In addition, using the scores of patients, pre- and post-operatively, graphs with differences were further developed to assess the degree of improvement. In this case, the pre-surgery score for each characteristic was subtracted from the corresponding score in the follow-up period. The greater the negative change in the score, the better the improvement of patients, i.e., reduction of symptoms. Such diagrams were constructed for sex, age and BMI.

Statistical comparisons. Statistical comparisons were made for all characteristics aforementioned in the descriptive statistics section. For all analyses, the level of significance was set to $5 \% ; \mathrm{P}<0.05$ was considered to indicate a statistically significant difference. Depending on the scale of the variables (e.g., nominal, ordinal, scale) and whether they followed normal distribution or not, the appropriate parametric or non-parametric methods were applied. For the group comparisons (e.g., between the two age groups or among the several BMI categories), independent group analyses were conducted 
using the symptom score value as dependent variable and the group categories as independent variables. Independent group analyses comprised of either the parametric methods, independent t-test and one-way ANOVA, or the non-parametric methods, Mann-Whitney and Kruskal-Wallis. When a multiple comparisons method (such as ANOVA) resulted in significant results, the post hoc group comparison methods of LSD and Tukey's test were applied. For the comparison of symptom values before and after surgery, the paired t-test or Wilcoxon signed rank test were used, for parametric and non-parametric comparisons, respectively. Comparison of proportions (namely, between two groups with count data) was made using the independent group comparison with the z-statistic.

Shapiro-Wilk, Kolmogorov-Smirnov and QQ plots were used to assess normal distribution of data. Exploration of association between two categorical variables (such as satisfaction level and surgery) was performed using $\chi^{2}$ analysis.

Data confidentiality. In the present study, patient information remained confidential in accordance with the General Data Protection Regulation rules (Regulation 2016/679 of the European Parliament 2016) (27). Patient names were kept confidential and remained confidential in any reproduction of the data. Immediately after data collection, the names were deleted from the input sheet and replaced with a unique number in a random manner so that the patient could not be identified. Any researcher other than the Director of the surgical team had to sign a confidentiality statement in order to handle the data with the names. A statement of confidentiality was not required of those who had access to the encrypted data.

\section{Results}

Demographics. Within a period of 10 years (2009-2019), 144 patients underwent the Nissen fundoplication technique. The median age of patients was 57.5 years, and an almost equal proportion of men and women participated in the study (Table I). The classification of patients according to their BMI revealed that the majority of them $(68 \%)$ were overweight and obese, whereas only a very low proportion were underweight $(0.7 \%)$ (Table I).

Medications. Regarding the medications received for treating GERD symptoms pre-operatively, PPIs were the most common; $86.8 \%$ of patients took PPIs for their symptoms (Table II). Among them, esomeprazole was the most frequently administered PPI, followed by omeprazole, pantoprazole, rabeprazole and lastly lansoprazole (Table III). The once-a-day administration was the most popular and was followed by $68 \%$ of patients, whereas only a small proportion received PPIs twice a day (Table III). Some patients had more than one daily dose regimen.

Pre-operative findings. The most common GERD symptoms, including heartburn, difficulty swallowing and reflux, as well as response to medication, were examined for all patients pre-operatively. In order to provide a more in-depth insight, these symptoms were assessed with regards to BMI (Fig. 1), age (Fig. 2) and sex (Fig. 3).

In the categorization based on BMI, most of the patients from all subcategories (normal weight, overweight and obese)
Table I. Characteristics of patients at baseline.

\begin{tabular}{lc}
\hline Characteristics & Number of patients (n \\
\hline Median age (IQR), years & $57.5(65-47.25)$ \\
Female sex, n (\%) & $68(47)$ \\
Male sex, n (\%) & $76(53)$ \\
BMI, n (\%) & \\
Underweight & $1(0.7)$ \\
Normal weight & $45(31.3)$ \\
Overweight & $62(43)$ \\
Obese & $36(25)$
\end{tabular}

BMI, body mass index.

Table II. Medicines used to treat gastroesophageal reflux disease symptoms before surgery.

\begin{tabular}{lc}
\hline Medication & $\mathrm{N}(\%)$ \\
\hline PPIs & $125(86.8)$ \\
$\mathrm{H}_{2}$ receptor antagonists & $9(6.3)$ \\
Antacids & $9(6.3)$ \\
\hline
\end{tabular}

PPIs, proton pump inhibitors.

Table III. PPI use; type of PPI and daily dose.

\begin{tabular}{ll}
\hline Variable & $\mathrm{N}(\%)$ \\
\hline PPI type & \\
Esomeprazole & $56(39)$ \\
Omeprazole & $23(16)$ \\
Pantoprazole & $20(14)$ \\
Rabeprazole & $16(11)$ \\
Lansoprazole & $10(7)$ \\
Daily dose of PPI & \\
Once a day & $98(68)$ \\
Twice a day & $20(14)$ \\
Occasionally & $13(9)$ \\
\hline
\end{tabular}

PPIs, proton pump inhibitors.

reported very intense heartburn and reflux that made their daily life difficult (Fig. 1A and D). Very limited data were available for underweight patients; thus, no conclusions were drawn from them. The response to their medication ranged from moderate to relatively high (Fig. 1C). However, for the majority of patients from all BMI categories, difficulty swallowing was not observed (Fig. 1B). In order to investigate whether age affected the exhibition of symptoms, the analysis continued with the construction of bar graphs, where patients were divided into two age groups: Under 55 and above 55 years old (Fig. 2). For heartburn, swallowing 

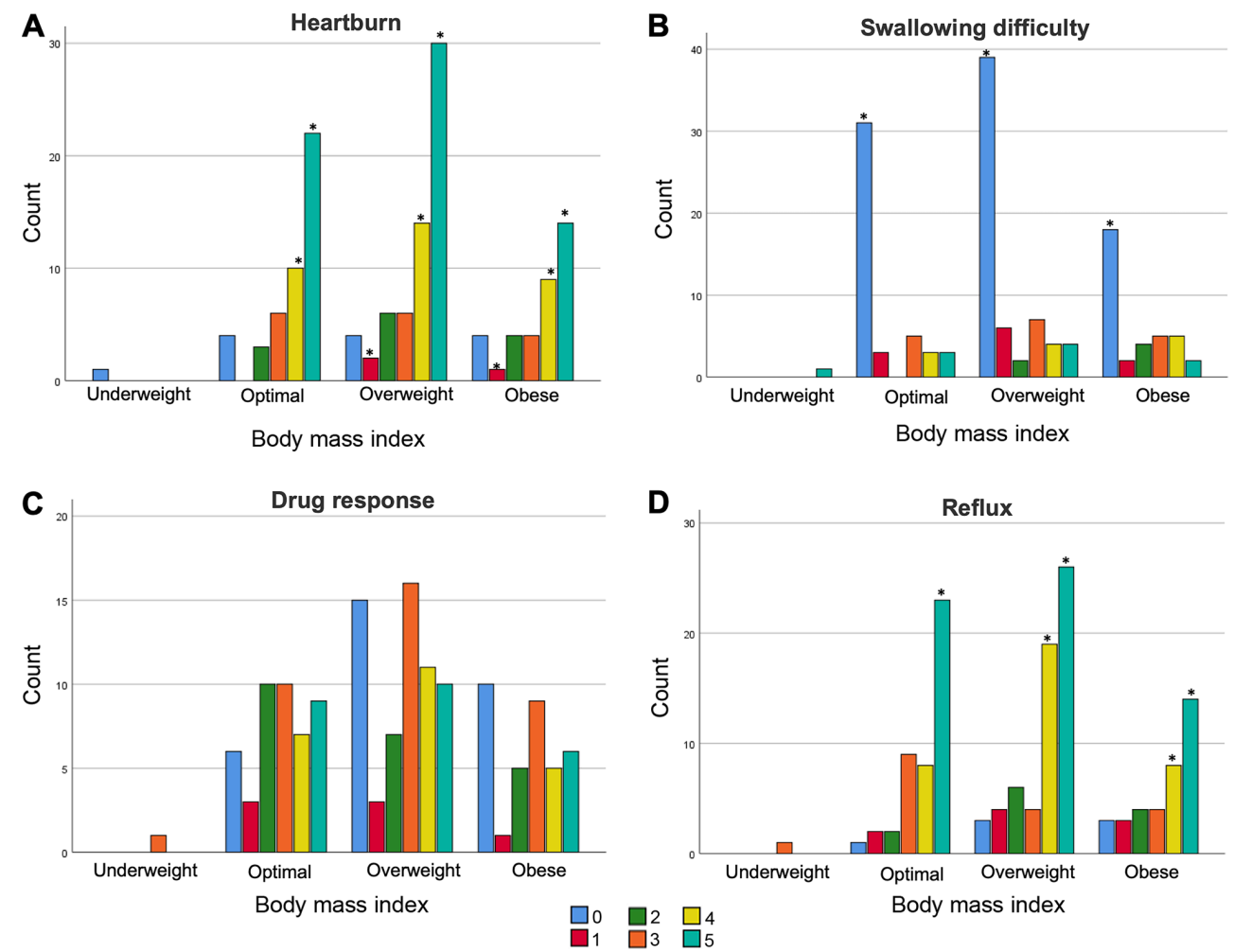

Figure 1. Intensity of gastroesophageal reflux disease symptoms before surgery, stratified by body mass index category (underweight, optimal, overweight, obese). (A) Heartburn, (B) difficulty swallowing, (C) relief of symptoms as a result of medication and (D) reflux. The intensity of symptoms was ranked from 0 (no symptoms) to 5 (highest intensity). "Intensity of symptom scores that differ statistically significant $(\mathrm{P}<0.05)$ within a BMI group. The proportions of symptom intensities were compared in pairs. The asterisks refer to an intensity group that differs from all other groups. A group without an asterisk may be different to another group, but not to all other groups.

A

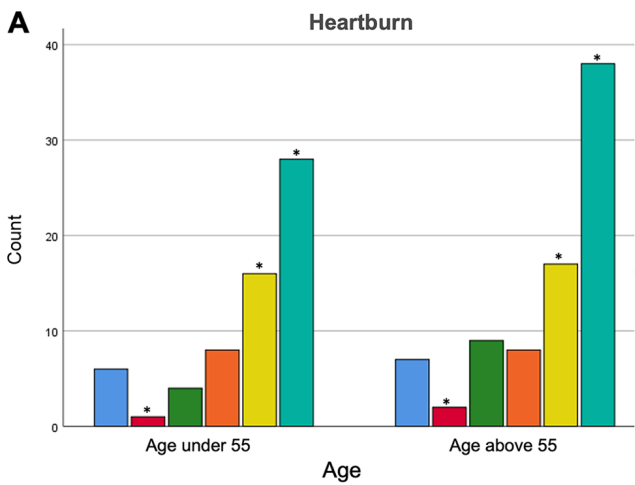

C

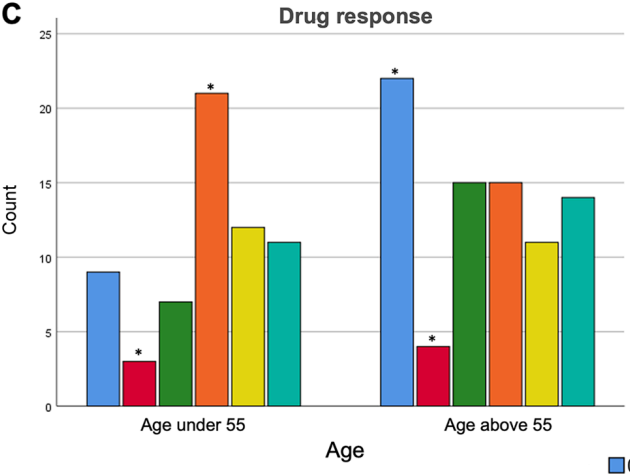

B

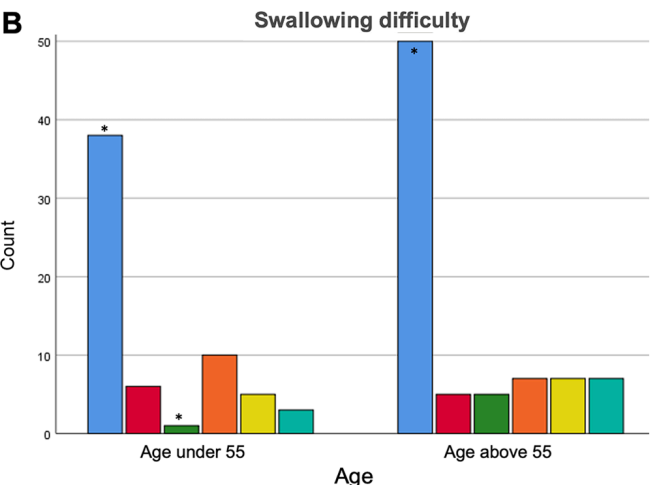

D

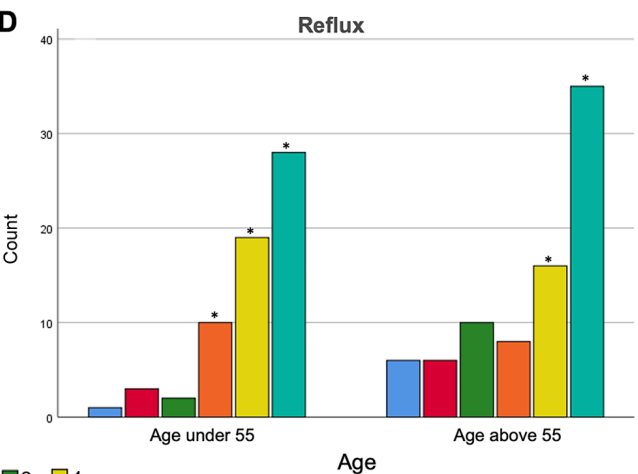

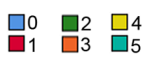

Figure 2. Intensity of gastroesophageal reflux disease symptoms before surgery stratified by age group (under 55 years or above 55 years). (A) Heartburn, (B) difficulty swallowing, (C) relief of symptoms as a result of medication and (D) reflux. The intensity of symptoms was ranked from 0 (no symptoms) to 5 (highest intensity). "Intensity symptom scores that differ statistically significant $(\mathrm{P}<0.05)$ within an age group. The proportions of symptom intensities were compared in pairs. The asterisks refer to an intensity group that differs from all other groups. A group without an asterisk may be different to another group, but not to all other groups. 
A

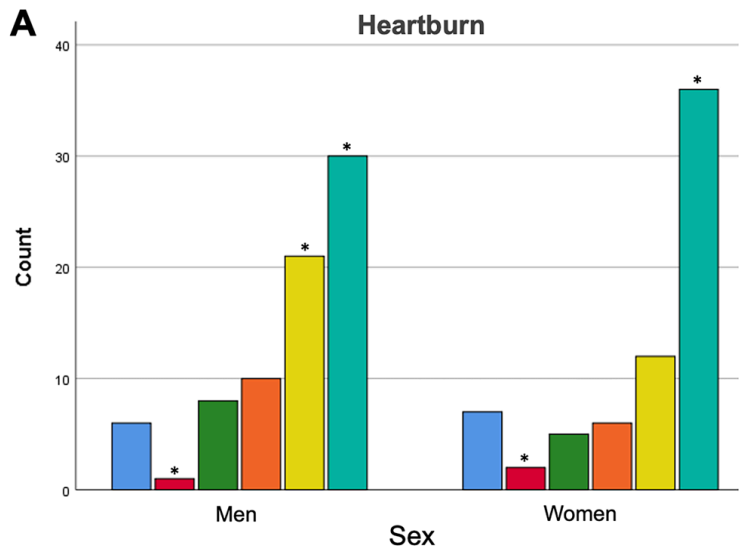

C

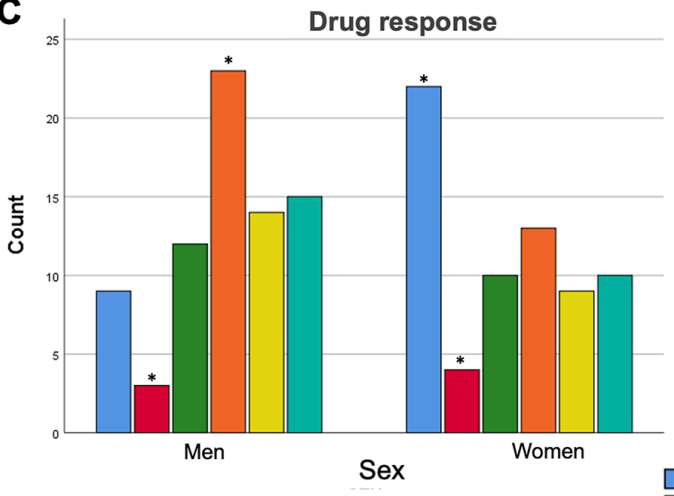

B

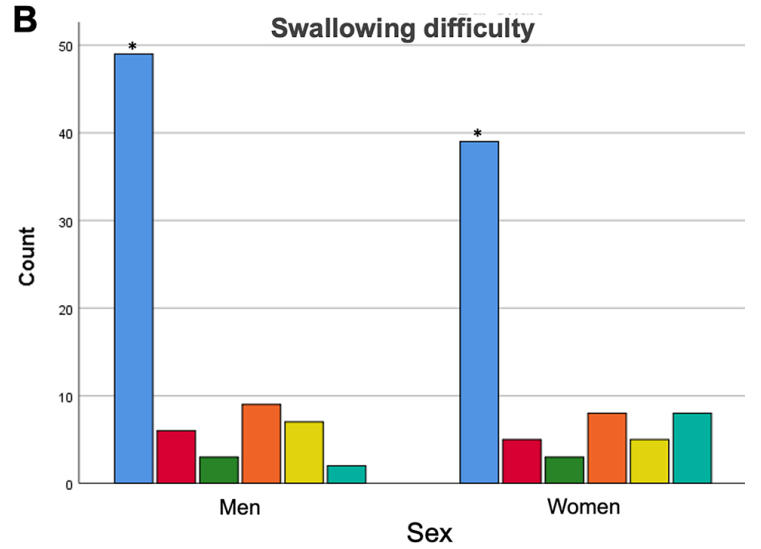

D

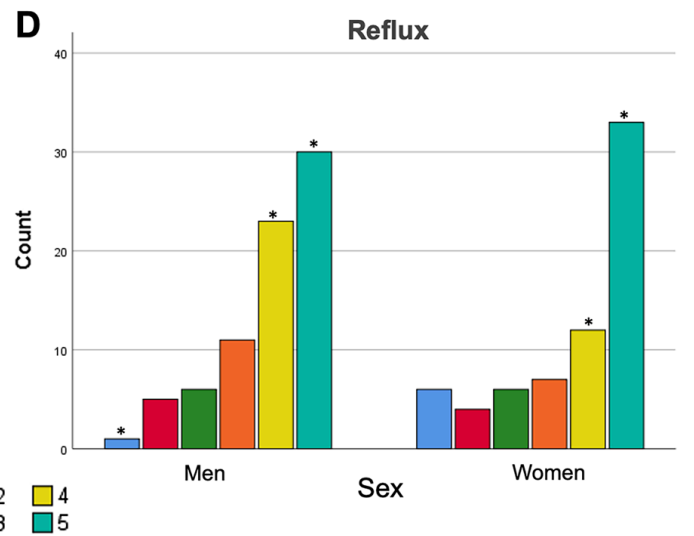

Figure 3. Intensity of gastroesophageal reflux disease symptoms before surgery stratified by sex. (A) Heartburn, (B) difficulty swallowing, (C) relief of symptoms as a result of medication and (D) reflux. The intensity of symptoms was ranked from 0 (no symptoms) to 5 (highest intensity). *Intensity symptom scores that differ statistically significant $(\mathrm{P}<0.05)$ within a sex group. The proportions of symptom intensities were compared in pairs. The asterisks refer to an intensity group that differs from all other groups. A group without an asterisk may be different to another group, but not to all other groups.

difficulty and reflux, a similar pattern was observed between the two age groups, with no large differences in symptoms between young and older patients (Fig. 2A, B and D). Most people experienced symptoms that interfered with their daily activity, followed by successively less intense symptoms. It appears that younger patients suffered more than older patients, since a much lower proportion in the younger group did not show symptoms (Fig. 2A, B and D), whereas patients in the $>55$ years group, had a poorer response to medication (Fig. 2C). The drug response in the $>55$ age group was moderate to deficient (Fig. 2C). With regards to statistical comparisons, ANOVA was applied among the BMI groups, whereas independent t-test was used to compare the two age groups and the two sex groups. In Fig. 1, no significant differences were revealed among the four BMI categories (ANOVA; $\mathrm{P}>0.05)$. Similar results were also observed for the comparison of scores between the two age groups in Fig. 2 (independent samples t-test; $\mathrm{P}>0.05$ ). The only marginal, but still not significant, differences referred to the difference in heartburn among the $\mathrm{BMI}$ groups $(\mathrm{P}=0.061)$ and in reflux, which was higher in the $>55$ years group $(\mathrm{P}=0.060)$.

Analysis between the sexes did not reveal statistical differences between men and women (Fig. 3). The present study revealed that $70.6 \%$ of women and $67.1 \%$ of men had heartburn that interfered with their daily activity (Fig. 3A). The majority of both sexes did not experience pain at swallowing (Fig. 3B), whereas reflux symptoms were quite similar between men and women; $69.7 \%$ of men and $66.2 \%$ of women exhibited severe reflux (Fig. 3D). Notably, $72 \%$ of women did not respond at all, or responded moderately, to their medication (Fig. 3C), whereas the relevant percentage (of moderate or no response) of men was $61.8 \%$.

When the patients were asked about their quality of life, the vast majority of them were dissatisfied with their daily life, irrespective of their BMI (Fig. 4A). Similar results were observed following the separation of patients into two groups based on their age; the vast majority of people in both age groups were not satisfied with their quality of life (Fig. 4B). Furthermore, no differences were identified in terms of sex; almost all patients reported being dissatisfied with their quality of life, irrespective of sex (Fig. 4C).

A more detailed presentation of the impact of BMI, age and sex on the various types and intensity of heartburn (e.g., discriminating when lying down, after meals etc.), swallowing pain and reflux is provided in Figs. S1-S9.

Post-operative changes. The present study subsequently compared the GERD-related symptoms before and after the operation, in order to assess the degree of improvement (Fig. 5). Notably, the feeling of heartburn improved rapidly. After surgery, only $20 \%$ of patients still suffered from heartburn (compared with $91 \%$ pre-operatively), $18 \%$ of people felt it during sleep and $16 \%$ when standing up, whereas the relevant percentages before surgery were $\sim 90 \%$. In addition, 

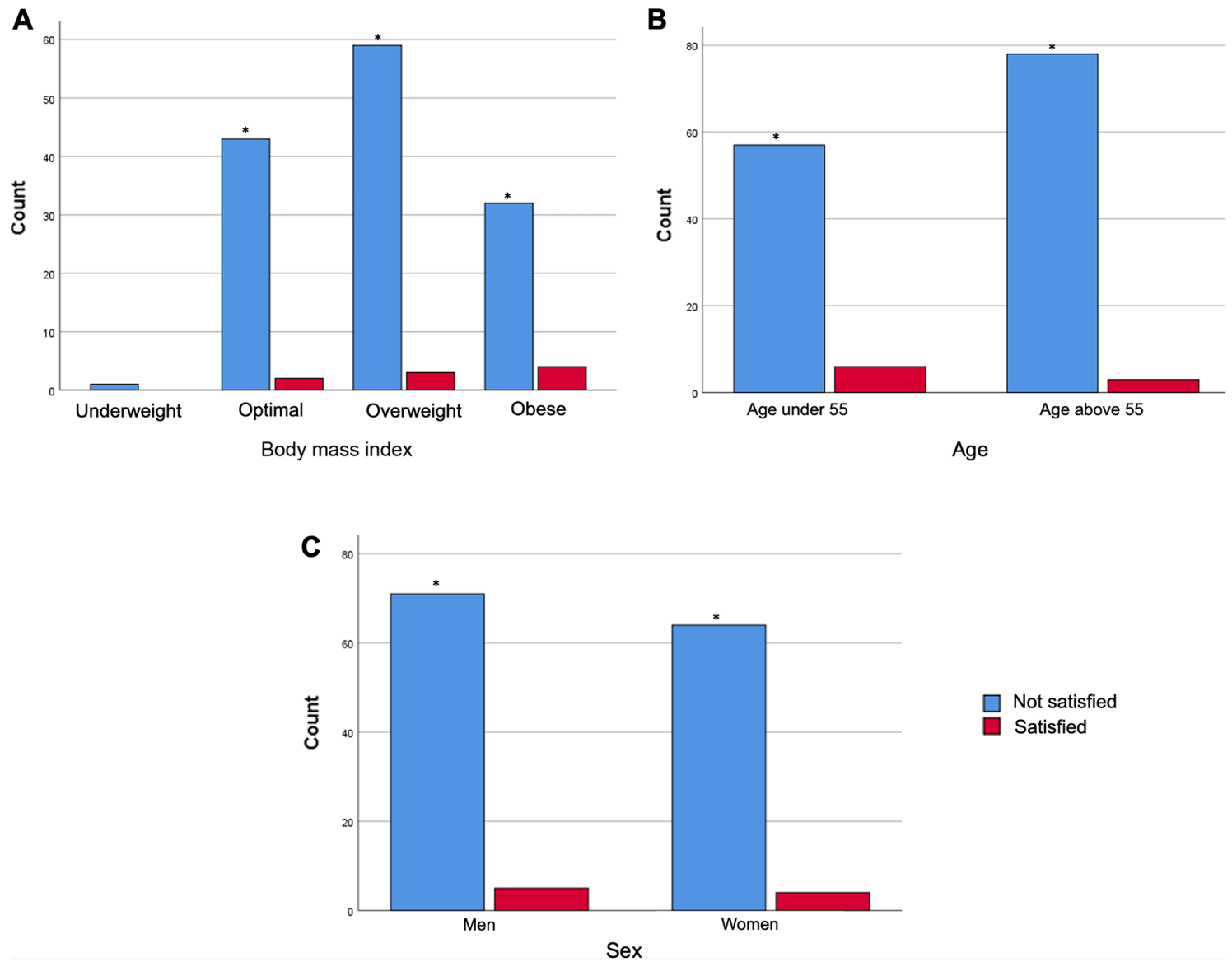

Figure 4. Satisfaction level of patients before surgery. Patients' opinion about their quality of life is stratified by (A) body mass index, (B) age group and (C) sex. "P $<0.05$ vs. satisfied group.

A

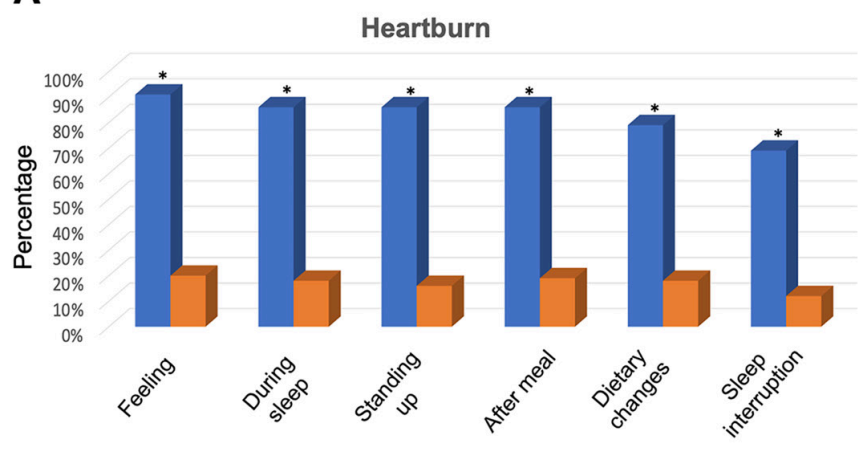

C

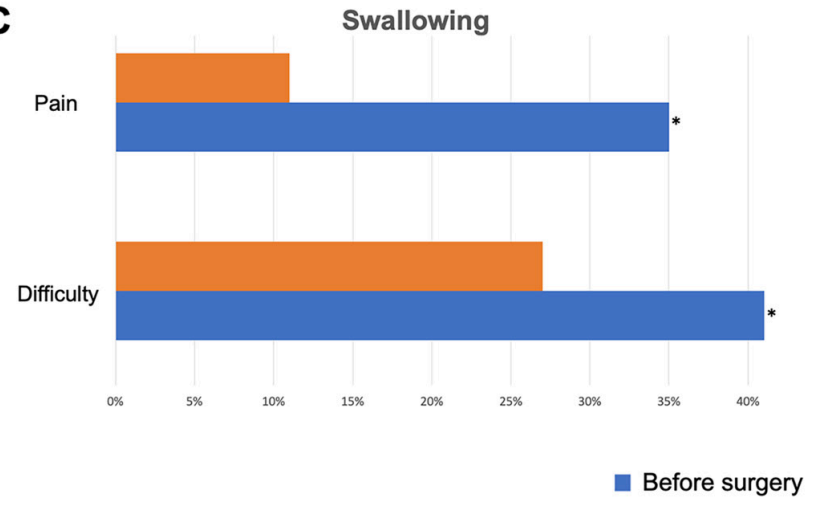

B

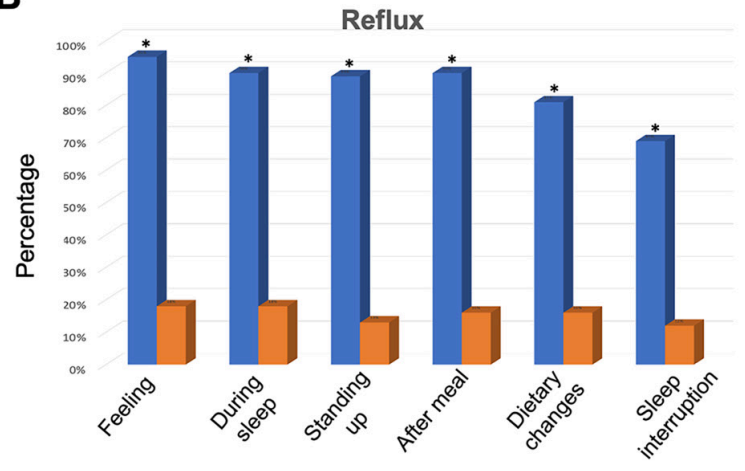

D

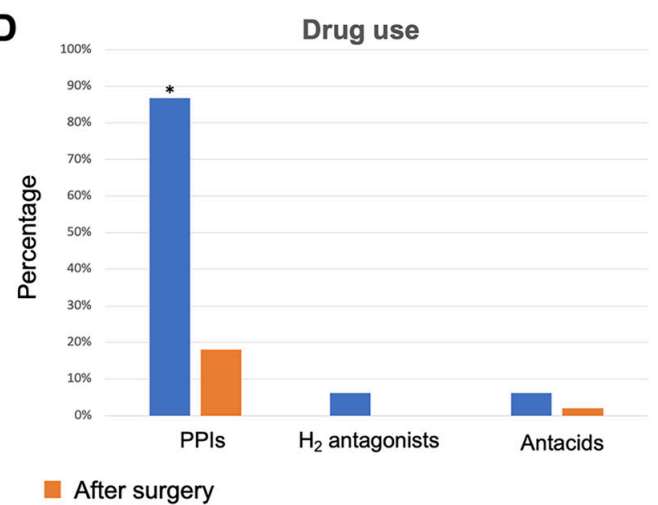

Figure 5. Proportion of patients exhibiting symptoms before and after surgery. The symptoms analyzed refer to (A) heartburn, (B) reflux, and (C) pain or difficulty swallowing before and after surgery. (D) Drug use before and after surgery is also shown. ${ }^{*} \mathrm{P}<0.05$ vs. after surgery. PPI, proton pump inhibitor. 


\section{A Satisfaction}

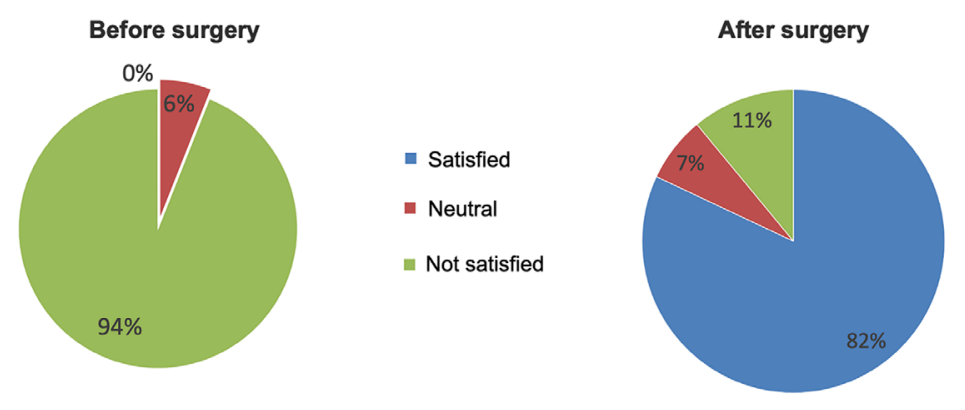

\section{B GERD total score}

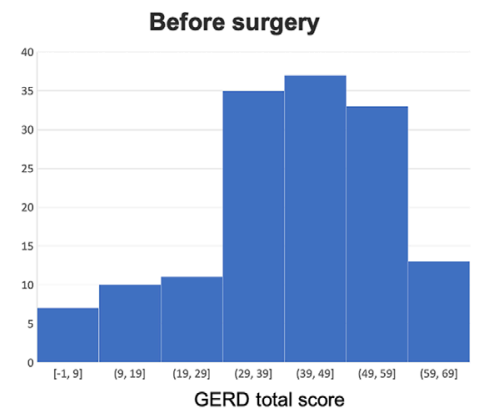

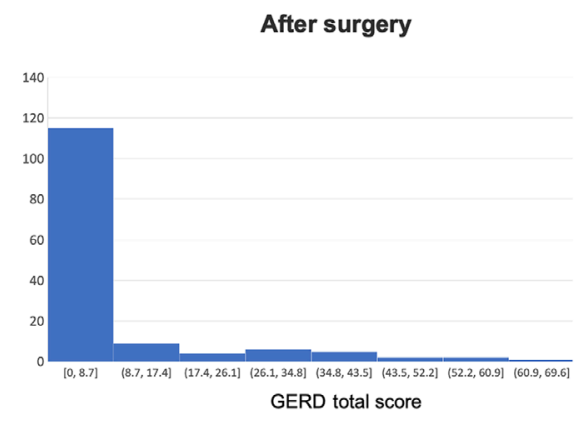

Figure 6. Satisfaction and GERD total score before and after surgery. (A) Patients were directly asked to answer their degree of 'satisfaction'. (B) The GERD total score was calculated from the relevant questionnaire using scores from numerous symptoms $(25)$. Statistically significant improvements $(\mathrm{P}<0.05)$ were observed after surgery. GERD, gastroesophageal reflux disease.

only $19 \%$ continued to feel heartburn after meals, $18 \%$ still needed to make dietary changes and $12 \%$ had a problem with sleep, compared with the pre-operative proportions of $\sim 80 \%$ (Fig. 5A). In addition, reflux improved just as markedly after surgery (Fig. 5B). After surgery, only $18 \%$ of patients experienced reflux, $18 \%$ felt it during bedtime and $13 \%$ when standing up, compared with the relevant percentages of $>90 \%$ pre-operatively. Similar pronounced improvements were observed in the need for dietary changes and sleep without interruption. Dysphagia and pain swallowing were also markedly improved post-operatively (Fig. 5C), whereas drug use was significantly reduced after surgery (Fig. 5D). Pre-operatively, the use of PPIs reached $86.8 \%$, and the proportion of patients using $\mathrm{H}_{2}$-antagonists and antacids was $6.8 \%$. However, post-operatively, none of the patients used $\mathrm{H}_{2}$-antagonists, whereas only $2 \%$ of them received antacids and $18 \%$ received a PPI (Fig. 5D).

Notably, the differences in all heartburn- and reflux-related symptoms (i.e., feeling, heartburn during sleep, when standing up, after a meal, and whether it leads to sleep interruption and the need for dietary changes), pain and difficulty at swallowing, and drug use before and after surgery were statistically significant $(\mathrm{P}<0.05$; Fig. 5).

The overall quality of life of patients was also assessed by recording their answer to the direct question of whether they were satisfied (with answers of: Yes, no and neutral); the results of this assessment are depicted in Fig. 6. Before surgery, the vast majority (94\%) of patients expressed their dissatisfaction about their everyday life, whereas the proportion was inversed after surgery; $82 \%$ of patients were satisfied, $7 \%$ were neutral and only $11 \%$ were not satisfied (Fig. 6A). The findings were further verified when the total GERD score was assessed. Pre-operatively, the GERD score was high, with an average estimate of $\sim 44$; however, after surgery, GERD score was minimized and the mean value was $\sim 4$ (Fig. 6B). This marked post-operative reduction in GERD score reflected the pronounced improvement of patients. $\chi^{2}$ analysis for the comparison of the relationship between satisfaction level and surgery (before vs. after; Fig. 6A), resulted in a significant difference $(\mathrm{P}<0.05)$. In addition, when Wilcoxon statistical test was applied to the pre- and post-operative GERD total score (Fig. 6B), a statistically significant difference was revealed (Table $\mathrm{SI}$ ).

Patients were also asked to characterize the change in how they felt before and after their operation. The extent of improvement was assessed with regards to BMI, age group and sex (Fig. 7). In all cases, the great majority of patients felt great improvement in their overall life conditions post-operatively. Much lower proportions of patients stated no or intermediate improvement. The same findings were observed in all situations regardless of BMI, whereas older patients exhibited greater improvement than younger patients. Also, even though women experienced more severe symptoms before surgery, they appeared to respond as well as the male patients after surgery. All types of heartburn scores, reflux scores, satisfaction level, pain and difficulty at swallowing scores, and total GERD score for each individual patient were compared before and after surgery using the Wilcoxon test. In all cases, statistically significant differences $(\mathrm{P}<0.05)$ were found before and after surgery (Table SI).

Since previous results indicated a clear improvement in all symptoms and the general condition of patients after surgery, the two most typical symptoms of heartburn and reflux were further assessed short-, mid- and long-term after surgery (Fig. 8). For 
A

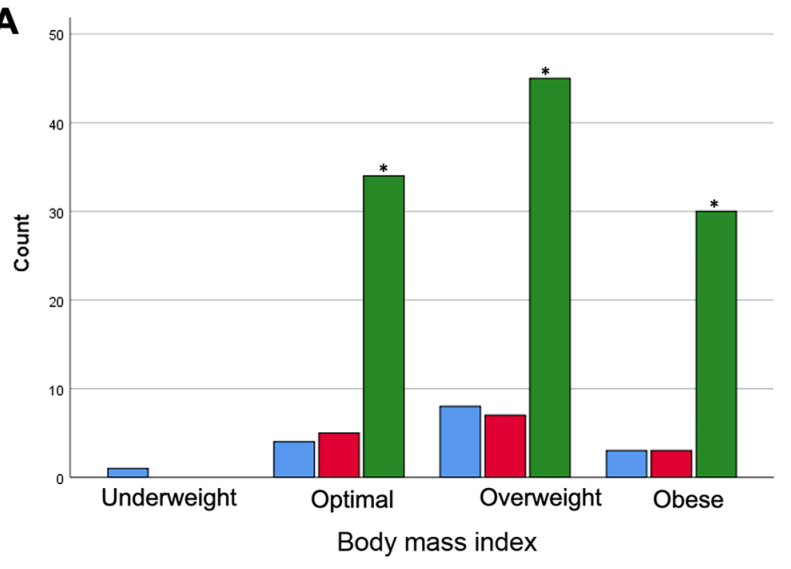

B

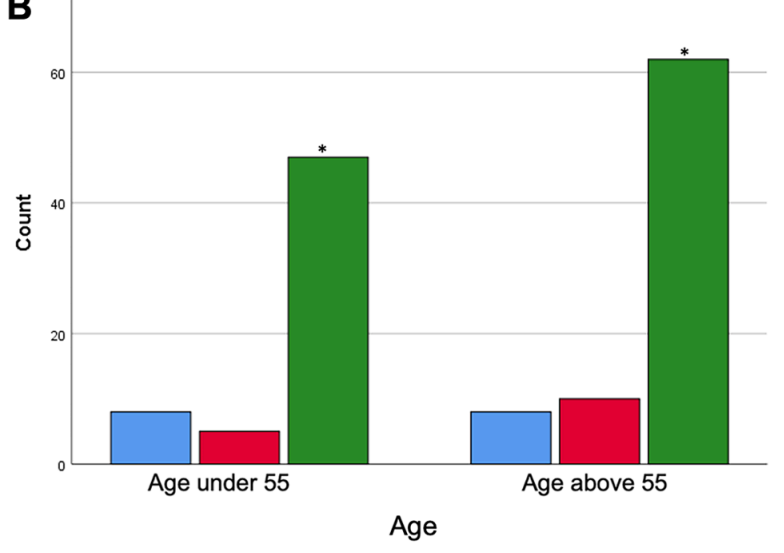

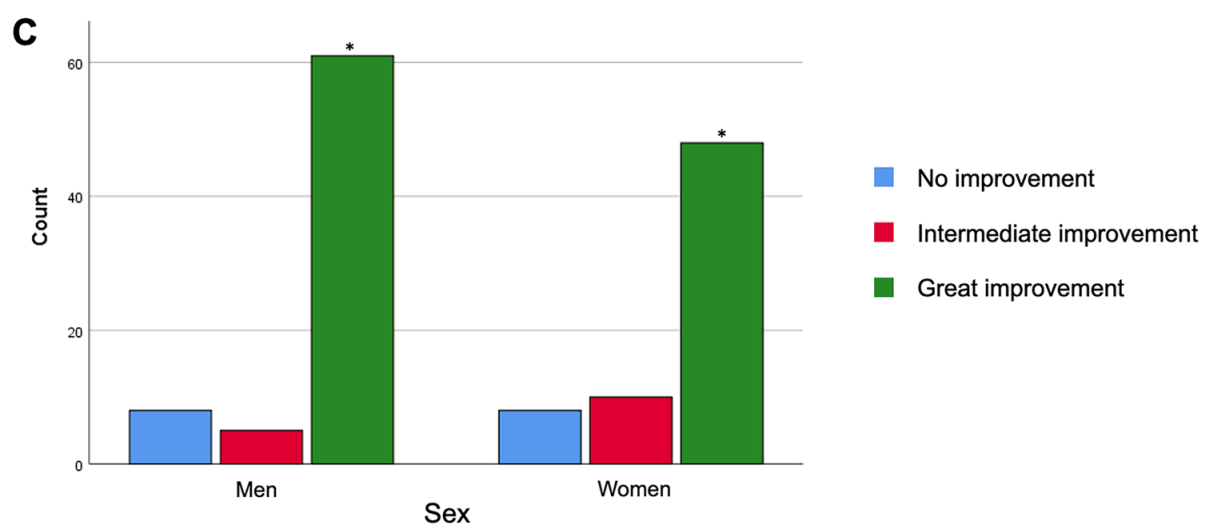

Figure 7. Extent of improvement after surgery. Improvement was analyzed with regard to (A) body mass index, (B) age group and (C) sex. ${ }^{*} \mathrm{P}<0.05$.

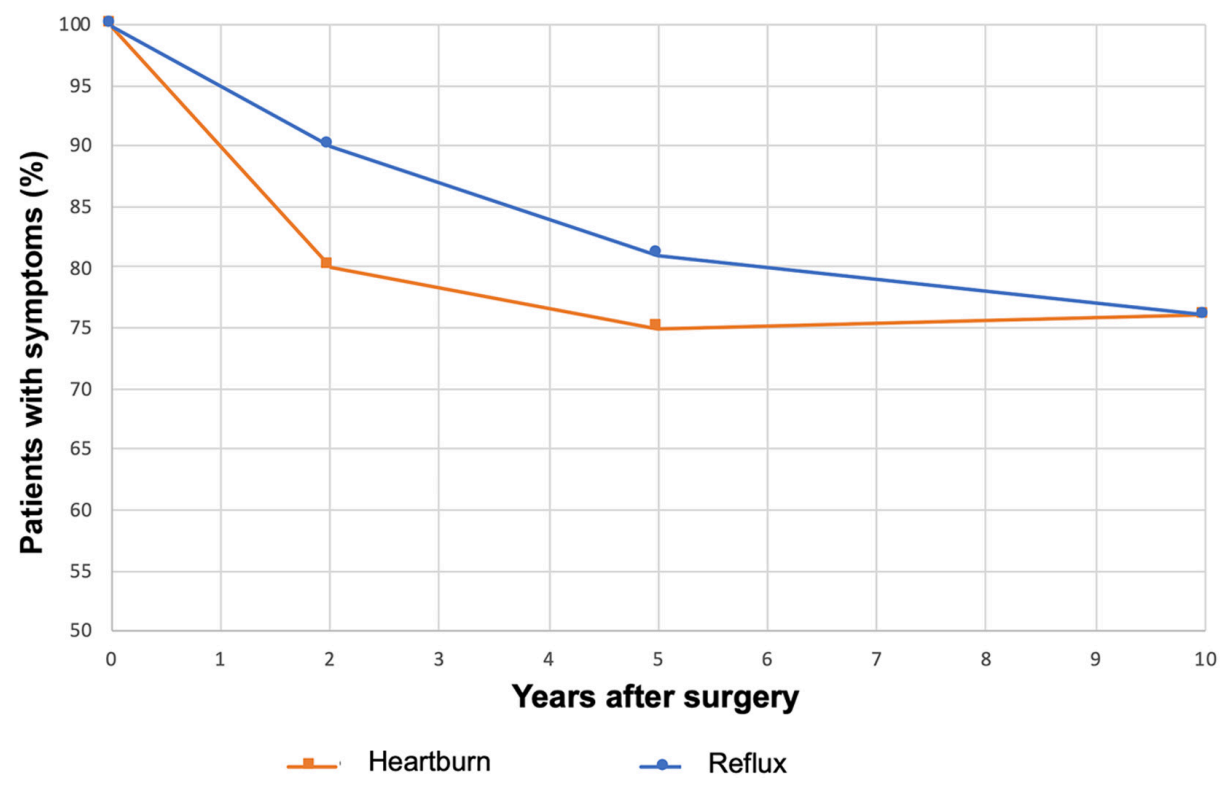

Figure 8. Post-operative short-, mid- and long-term heartburn and reflux. Percentage of patients still exhibiting symptoms compared to those having symptoms immediately after surgery.

those patients who still experienced any level of heartburn and reflux post-operatively, the evolution of symptoms was studied over time. It was revealed that, as time passes, the condition of patients improved, since the percentages of those who still felt heartburn or reflux were lower (Fig. 8). These findings indicated that a marked improvement was observed post-operatively and the situation may become even better as time passes. The long-term effectiveness of surgery was thus confirmed, even if several years had passed.

A more detailed description of the effects of BMI, age and sex on the differences in intensity of heartburn, difficulty swallowing and reflux is provided in Figs. S10-S12. 
With regards to post-operative complications, post-operative hypoxemia, blood loss and length of hospital stay were investigated. A total of $95 \%$ of patients stayed only 1 day in the hospital, whereas the remaining 5\% stayed for 2 days. None of the operated patients exhibited hypoxemia and no one was transferred to the intensive care unit. In addition, there was minimal blood loss (ca. $20 \mathrm{ml}$ ) in all of these GERD operations.

\section{Discussion}

In the present study, the quality of life of patients suffering from GERD was compared pre-operatively, when the patients received medication to treat their symptoms, and post-operatively, in order to assess the beneficial effect of surgery. The study took place at the Athens Medical Center, between October 2019 and March 2020. A total of 144 patients (men and women) who were suffering from GERD were recruited to the present study and symptoms were evaluated post-surgery.

Based on the scores obtained from the questionnaires, data were analyzed in terms of BMI, age group and sex. The majority of patients in each category experienced intense heartburn and reflux symptoms. Notably, younger patients suffered more than older patients; however, patients in the $>55$ years group, had a poorer response to medication. In addition, women appeared to have more severe symptoms and a reduced response to medications compared with men. Finally, quality of life, as specified by the patients themselves, was extremely low regardless of weight, sex and age.

The patients were re-examined after surgery and it was revealed that the symptoms of heartburn and reflux were markedly reduced. The majority of patients in each BMI category were almost completely cured and a rapid improvement in quality of life was observed. Pre-operatively, none of the patients expressed satisfaction, whereas after the operation the percentage of satisfied patients reached $82 \%$. This finding is in line with other studies reporting a relevant improvement in living standards $(28,29)$. Regarding the age of the patients, the present study demonstrated that older patients exhibited better cure rates, implying a relationship between the age of the patients and the success of the surgery. This finding is in contrast to a previous study, in which no similar relationship was found (30).

In order to assess the long-term beneficial effects of surgery, the effects of the robotic operation were studied through the years. The desired post-operative improvement in heartburn and reflux was maintained and the findings indicated that the situation became slightly better over time. However, that possible improvement over time was not found to be significant $(\mathrm{P}=0.196$, after comparing two subsequent population proportions with the $\mathrm{z}$-score). Thus, the long-term effectiveness of surgery was confirmed for up to 10 years after surgery. Arcerito et al (31), who performed total fundoplication in the majority of the 50 patients recruited, revealed that the effect of surgery on dysphagia symptoms over a 30-month median follow-up, led to very improved patient outcomes, without mesh-related complications; a finding which was further validated over a longer time period in the present study. It should be clarified that in the present study, not all 144 patients were followed post-operatively for 10 years. This implies that each patient was uniquely classified to the short-, mid- and long-term group simply by taking into account the date of his/her surgery at the time the present study was conducted. Considering a stable rate of operations over these years, the number of patients falling into each category was almost the same.

The present study also assessed the medications administered to treat GERD. The first-line treatment of GERD symptoms are drugs that modify the $\mathrm{pH}$ of the stomach, such as PPIs. In the present study, PPIs were revealed to be the predominant medication received by the patients $(86.8 \%)$; among them, esomeprazole was the most commonly used. After surgery, only $18 \%$ of patients made occasional use of a PPI and only $2 \%$ used antacids. These findings are in line with other studies showing that low proportions of patients use PPIs 10 years after surgery, whereas medication use after surgery is a poor indicator of recurrent GERD $(28,29)$. This is in contrast with other studies reporting significantly higher rates of PPI use post-operatively $(25,32)$.

The objective of the present study was to assess the symptoms (heartburn, dysphagia and reflux) of patients with GERD and the administered medications, and to evaluate their quality of life before and after robotic surgery using the Nissen fundoplication technique. A retrospective study was conducted on 144 patients who underwent surgery and their data were statistically analyzed. The potential impact of BMI, age and sex were further investigated. All patients involved in this analysis were exhibited pre-operatively severe symptoms of heartburn, reflux and low quality of life. All of these symptoms were re-examined after the surgery, where a marked decrease was observed in their frequency and intensity. Post-operative patient satisfaction and quality of life were very high, and more than $4 / 5$ of patients declared high satisfaction. Improvement was not found to be affected by BMI, whereas older patients exhibited relatively greater improvement compared to younger people. Although women initially experienced more severe symptoms before surgery, they appeared to respond as well to surgery as male patients. The long-term beneficial effect of robotic surgery for up to the 10 -year period studied was validated. Additional randomized clinical trials, using increased sample sizes, are required to verify these interesting findings.

\section{Acknowledgements}

Not applicable.

\section{Funding}

No funding was received.

\section{Availability of data and materials}

The datasets generated and/or analyzed during the current study are not publicly available due to the confidentiality of clinical data but are available from the corresponding author on reasonable request.

\section{Authors' contributions}

SKK, VK and KK conceived and designed the experiments. PK and GEA performed the experiments. PK, GEA, and KK 
confirmed authenticity of raw data. PK and GEA drafted the manuscript. SKK, PK, GEA, SLM, VK and KK finalized the manuscript. SKK supervised the experiments. PK, GEA and VK performed data analysis and interpretation. SKK, SLM and $\mathrm{KK}$ verified the results of the experiments, helped with the statistical analysis and revised the manuscript critically for intellectual content. All authors read and approved the final manuscript.

\section{Ethics approval and consent to participate}

The present study was approved by the Scientific Committee of the Athens Medical Center. All patients provided oral consent before providing their answers to the questionnaire.

\section{Patient consent for publication}

Not applicable.

\section{Competing interests}

The authors declare that they have no competing interests.

\section{References}

1. Mittal RK: Pathophysiology of gastroesophageal reflux disease: Motility factors. In: The Esophagus. Castell DO and Richter J (eds). Lippincott Williams \& Wilkins, Philadelphia,PA, pp407-420, 2004.

2. Diener U, Patti MG, Molena D, Fisichella PM and Way LW: Esophageal dysmotility and gastroesophageal reflux disease. J Gastrointest Surg 5: 260-265, 2001.

3. El-Serag HB, Sweet S, Winchester CC and Dent J: Update on the epidemiology of gastro-oesophageal reflux disease: A systematic review. Gut 63: 871-880, 2014.

4. Bredenoord AJ, Weusten BL, Curvers WL, Timmer R and Smout AJ: Determinants of perception of heartburn and regurgitation. Gut 55: 313-318, 2006.

5. Katz PO, Gerson LB and Vela MF: Guidelines for the diagnosis and management of gastroesophageal reflux disease. Am J Gastroenterol 108: 308-328, quiz 329, 2013.

6. Irwin RS, French CL, Curley FJ, Zawacki JK and Bennett FM Chronic cough due to gastroesophageal reflux. Clinical, diagnostic, and pathogenetic aspects. Chest 104: 1511-1517, 1993.

7. Richter JE, Pandolfino JE, Vela MF, Kahrilas PJ, Lacy BE, Ganz R, Dengler WB, Oelschlager PJ, DeVault KR, Fass R, et al; Esophageal Diagnostic Working Group: Utilization of wireless $\mathrm{pH}$ monitoring technologies: a summary of the proceedings from the esophageal diagnostic working group. Dis Esophagus 26: 755-65, 2013.

8. Kaltenbach T, Crockett S and Gerson LB: Are lifestyle measures effective in patients with gastroesophageal reflux disease? An evidence-based approach. Arch Intern Med 166: 965-971, 2006.

9. Ness-Jensen E and Lagergren J: Tobacco smoking, alcohol consumption and gastro-oesophageal reflux disease. Best Pract Res Clin Gastroenterol 31: 501-508, 2017.

10. Hunt R, Armstrong D, Katelaris P, Afihene M, Bane A, Bhatia S, Chen MH, Choi MG, Melo AC, Fock KM, et al; Review Team: World Gastroenterology Organisation Global Guidelines: GERD global perspective on gastroesophageal reflux disease. J Clin Gastroenterol 51: 467-478, 2017.

11. Bou Daher H and Sharara AI: Gastroesophageal reflux disease, obesity and laparoscopic sleeve gastrectomy: The burning questions. World J Gastroenterol 25: 4805-4813, 2019.

12. Mejía-Rivas MA, Herrera-López A, Hernández-Calleros J, Herrera MF and Valdovinos MA: Gastroesophageal reflux disease in morbid obesity: The effect of Roux-en-Y gastric bypass. Obes Surg 18: 1217-1224, 2008.

13. Gu L, Chen B, Du N, Fu R, Huang X, Mao F, Khadaroo PA and Zhao S: Relationship between bariatric surgery and gastroesophageal reflux disease: A systematic review and meta-analysis. Obes Surg 29: 4105-4113, 2019.
14. Newberry $\mathrm{C}$ and Lynch K: The role of diet in the development and management of gastroesophageal reflux disease: Why we feel the burn. J Thorac Dis 11 (Suppl 12): S1594-S1601, 2019.

15. Moraes-Filho JP, Navarro-Rodriguez T, Barbuti R, Eisig J, Chinzon D and Bernardo W; Brazilian Gerd Consensus Group: Guidelines for the diagnosis and management of gastroesophageal reflux disease: An evidence-based consensus. Arq Gastroenterol 47: 99-115, 2010.

16. Khan BA, Sodhi JS, Zargar SA, Javid G, Yattoo GN, Shah A, Gulzar GM and Khan MA: Effect of bed head elevation during sleep in symptomatic patients of nocturnal gastroesophageal reflux. J Gastroenterol Hepatol 27: 1078-1082, 2012.

17. MacFarlane B: Management of gastroesophageal reflux disease in adults: A pharmacist's perspective. Integr Pharm Res Pract 7: 41-52, 2018.

18. Freedberg DE, Kim LS and Yang YX: The risks and benefits of long-term use of proton pump inhibitors: Expert review and best practice advice from the American Gastroenterological Association. Gastroenterology 152: 706-715, 2017.

19. Thomson AB, Sauve MD, Kassam N and Kamitakahara H: Safety of the long-term use of proton pump inhibitors. World J Gastroenterol 16: 2323-2330, 2010.

20. Haastrup PF, Thompson W, Søndergaard J and Jarbøl DE: Side effects of long-term proton pump inhibitor use: A review. Basic Clin Pharmacol Toxicol 123: 114-121, 2018.

21. Cheung KS and Leung WK: Long-term use of proton-pump inhibitors and risk of gastric cancer: A review of the current evidence. Therap Adv Gastroenterol: Mar 11, 2019 (Epub ahead of print). doi: 10.1177/1756284819834511.

22. Hartmann J, Jacobi CA, Menenakos C, Ismail M and Braumann C: Surgical treatment of gastroesophageal reflux disease and upside-down stomach using the Da Vinci robotic system. A prospective study. J Gastrointest Surg 12: 504-509, 2008.

23. Ceccarelli G, Romano A, Esposito G, De Rosa M, Bugiantella W, Miranda E, Fontani A and D'Andrea V: Robot-assisted Toupet fundoplication and associated cholecystectomy in symptomatic giant hiatal hernia with situs viscerum inversus - A case report and literature review. Int J Surg Case Rep 60: 371-375, 2019.

24. Georgiou E, Schoina E, Markantonis SL, Karalis V, Athanasopoulos PG, Chrysoheris P, Antonakopoulos F and Konstantinidis K: Laparoscopic total extraperitoneal inguinal hernia repair: Retrospective study on prosthetic materials, postoperative management, and quality of life. Medicine (Baltimore) 97: e13974, 2018

25. Castelijns PS, Ponten JE, Vd Poll MC, Bouvy ND and Smulders JF: Quality of life after Nissen fundoplication in patients with gastroesophageal reflux disease: Comparison between long- and short-term follow-up. J Minim Access Surg 14: 213-220, 2018.

26. Mahawar KK: Defining short-term, medium-term, long-term, and very long-term follow-up after bariatric surgery. Obes Surg 28: 1425-1426, 2018.

27. Regulation (EU) 2016/679 of the European Parliament and of the Council of 27 April 2016 on the protection of natural persons with regard to the processing of personal data and on the free movement of such data, and repealing Directive 95/46/EC. OJEU 119: 1-88, 2016

28. Dallemagne B, Weerts J, Markiewicz S, Dewandre JM, Wahlen C, Monami B and Jehaes C: Clinical results of laparoscopic fundoplication at ten years after surgery. Surg Endosc 20: 159-165, 2006

29. Morgenthal CB, Shane MD, Stival A, Gletsu N, Milam G, Swafford V, Hunter JG and Smith CD: The durability of laparoscopic Nissen fundoplication: 11-year outcomes. J Gastrointest Surg 11: 693-700, 2007.

30. Lee TG, Lee IS, Kim BS, Jung HY and Choi YB: Surgical outcomes and over one-year follow-up results of laparoscopic Nissen fundoplication for gastroesophageal reflux disease: Single-center experiences. Asian J Surg 42: 557-562, 2019.

31. Arcerito M, Changchien E, Falcon M, Parga MA, Bernal O and Moon JT: Robotic fundoplication for gastroesophageal reflux disease and hiatal hernia: Initial experience and outcome. Am Surg 84: 1945-1950, 2018.

32. Spechler SJ, Lee E, Ahnen D, Goyal RK, Hirano I, Ramirez F, Raufman JP, Sampliner R, Schnell T, Sontag S, et al: Long-term outcome of medical and surgical therapies for gastroesophageal reflux disease: Follow-up of a randomized controlled trial. JAMA 285: 2331-2338, 2001.

This work is licensed under a Creative Commons Attribution-NonCommercial-NoDerivatives 4.0 International (CC BY-NC-ND 4.0) License. 